\title{
High-sensitivity noncontact atomic force microscope/scanning tunneling microscope (nc AFM/STM) operating at subangstrom oscillation amplitudes for atomic resolution imaging and force spectroscopy
}

\author{
A. Oral, ${ }^{a), b)}$ R. A. Grimble, H. Ö. Özer, ${ }^{\text {b) }}$ and J. B. Pethica \\ Department of Materials, University of Oxford, Parks Road, Oxford OX1 3PH, United Kingdom
}

(Received 27 August 2002; accepted 27 May 2003)

\begin{abstract}
We describe a new, highly sensitive noncontact atomic force microscope/scanning tunneling microscope (STM) operating in ultrahigh vacuum (UHV) with subangstrom oscillation amplitudes for atomic resolution imaging and force-distance spectroscopy. A novel fiber interferometer with $\sim 4 \times 10^{-4} \AA / \sqrt{\mathrm{Hz}}$ noise level is employed to detect cantilever displacements. Subangstrom oscillation amplitude is applied to the lever at a frequency well below the resonance and changes in the oscillation amplitude due to tip-sample force interactions are measured with a lock-in amplifier. Quantitative force gradient images can be obtained simultaneously with the STM topography. Employment of subangstrom oscillation amplitudes lets us perform force-distance measurements, which reveal very short-range force interactions, consistent with the theory. Performance of the microscope is demonstrated with quantitative atomic resolution images of $\mathrm{Si}(111)(7 \times 7)$ and forcedistance curves showing short interaction range, all obtained with $<0.25 \AA$ lever oscillation amplitude. Our technique is not limited to UHV only and operation under liquids and air is feasible.
\end{abstract} (C) 2003 American Institute of Physics. [DOI: 10.1063/1.1593786]

\section{INTRODUCTION}

At its invention, the atomic force microscope (AFM) (Ref. 1) was intended to be an analogue of the scanning tunneling microscope (STM), using the forces rather than the tunnel current between tip and surface atoms to generate an image. In principle, this should also give atomic resolution because short-range bonds such as covalent, have, like tunnel current, an exponential dependence on distance. ${ }^{2,3}$ The rapidity of exponential decay is generally accepted to be the reason for the high resolution. However, the actual development and wide applicability of AFM has mostly been at poorer resolution than atomic, mainly because of the extreme sensitivity required to resolve single bonds and the instability of soft, sensitive levers against high force gradients of shortrange interactions. Atomic resolution AFM has been achieved using resonant cantilevers with large, $\sim 100 \AA$, oscillation amplitudes in UHV. ${ }^{4-6}$ This technique is also used to deduce the force interactions between tip and sample, but large oscillation amplitudes employed are vastly greater than the interaction range and the interaction has to be deduced by using a deconvolution in which assumptions such as single valuedness, no loss, etc., have to be made. ${ }^{7}$ Durig et al. ${ }^{8}$ measured the short range interactions using an Ir sample for the first time, but this work did not involve any imaging. Other attempts ${ }^{9,10}$ for direct observations of the form of the bonding interaction have so far shown unexpectedly large length scales, which are incompatible with a true strong atomic bond. ${ }^{11}$

\footnotetext{
a) Author to whom correspondence should be addressed; electronic mail: ahmet@fen.bilkent.edu.tr

${ }^{b}$ Present address: Department of Physics, Bilkent University, 06533 Ankara, Turkey.
}

In this article, we describe a new combined noncontact AFM (nc-AFM) and STM that can operate at subangstrom oscillation amplitudes using stiff $(k \sim 100 \mathrm{~N} / \mathrm{m})$ cantilevers below resonance, which would allow quantitative atomic resolution imaging and force-distance spectroscopy. Use of sub- $\AA$ lever oscillation amplitudes simplifies the data analysis and allows direct determination of the interaction potential without the need for deconvolution of data from resonance shifts. ${ }^{7,12,13}$ Quantitative values of the bond stiffness values during imaging of $\mathrm{Si}(111)(7 \times 7)$ are thereby obtained. ${ }^{14}$ Furthermore, our technique operates in the liquids ${ }^{15}$ without loss of any sensitivity and has potential to achieve atomic resolution in liquids and in air.

\section{INSTRUMENTATION}

\section{A. UHV system}

The microscope is installed in a small, custom-designed ultra-high-vacuum (UHV) chamber, as shown in Fig. 1, which is equipped with a low-energy electron diffraction (LEED)/Auger ${ }^{16}$ system for sample analysis, argon sputtering gun and a home made electron beam heater for sample/ lever preparation. The system volume and surface area have been kept very small to achieve a low base pressure in the system. The main chamber is pumped with a combination pump, which is composed of a $300 \mathrm{l} / \mathrm{s}$ (Ref. 17) triode pump and a titanium sublimation pump. A small, 70 1/s turbomolecular pump ${ }^{18}$ backed with a double stage rotary pump is used to evacuate the fast entry lock for lever/sample transfer. An 8 in. travel linear-rotary motion feedthrough is employed as sample/lever manipulator. After baking the system out at $165^{\circ} \mathrm{C}$, a base pressure of $\sim 7 \times 10^{-11}$ mbar is routinely 


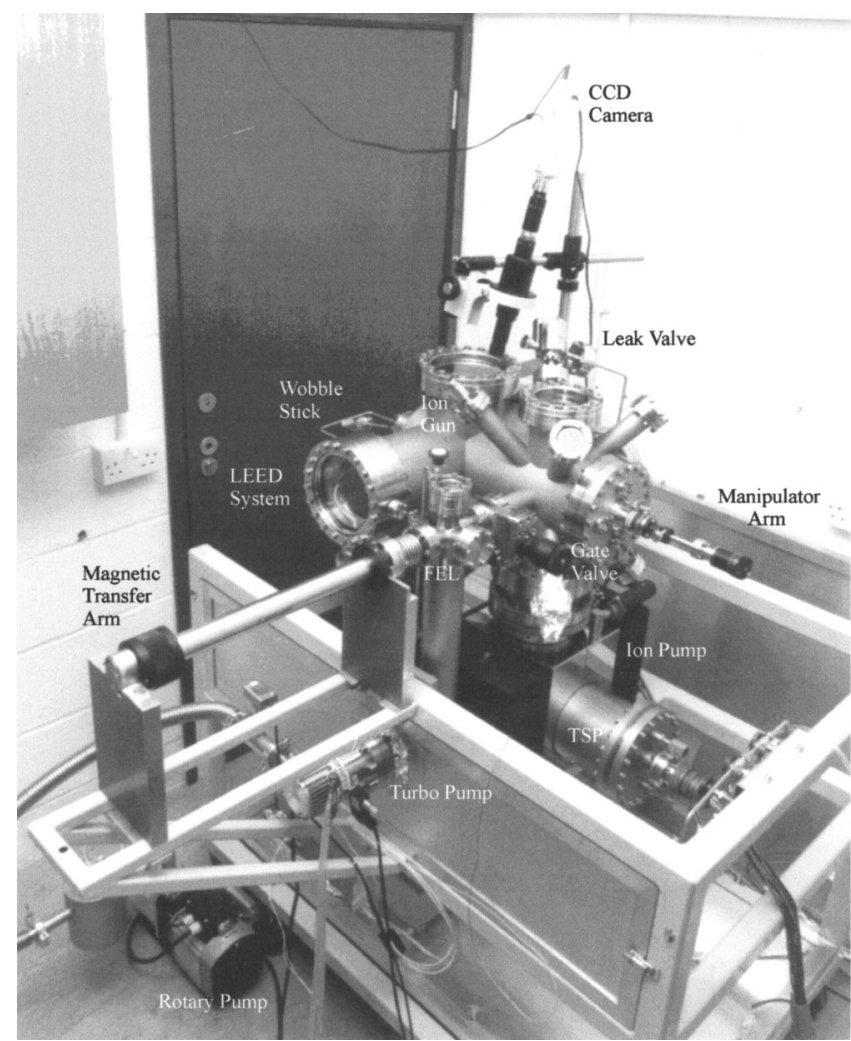

FIG. 1. Picture of the UHV system.

achieved. Atomically clean $\mathrm{Si}(111)(7 \times 7)$ is prepared in situ by standard methods finally flashing the sample to $1000^{\circ} \mathrm{C}$.

\section{B. NC-AFM/STM}

Our microscope is built on a special 8-in.-diam conflate UHV flange as shown in the Fig. 2. The design allows us to exchange levers and samples without breaking the vacuum through the load lock. Up to four samples/levers can be stored in a carousel attached to the microscope, which also acts as sample holder for LEED/Auger analysis. For vibration isolation, microscope base is suspended from four posts by using stainless steel springs and $\mathrm{Sm}-\mathrm{Co}$ magnet-copper sheet pairs are used for eddy current damping. This configuration has a natural frequency of $\sim 2 \mathrm{~Hz}$ and is sufficient to eliminate the external vibrations in our laboratory, which is located at the first floor. During the lever/sample transfer, the microscope base and the sample slider are locked to the four posts by a special mechanism actuated with a linear motion

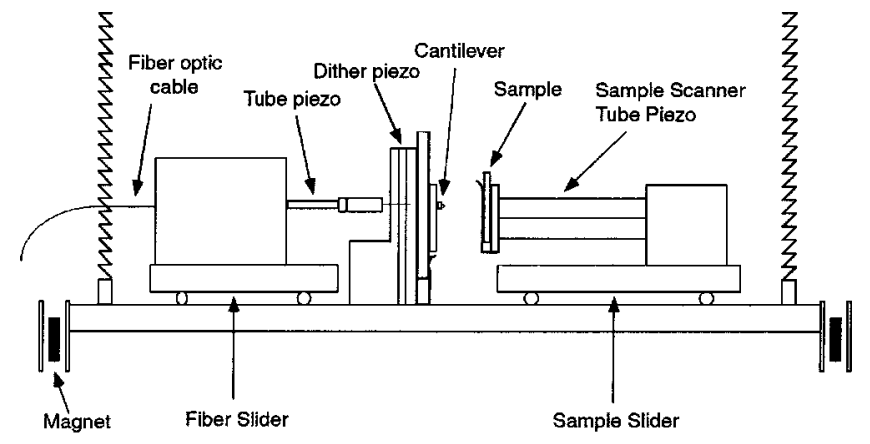

FIG. 2. Schematics of AFM/STM. feedthrough. A pincer-grip wobble stick $^{19}$ is used to exchange the levers/samples. Samples are held on tantalum holders by spot welded thin tantalum clips for direct electron beam heating. There is a hole at the sample holder where the sample sits, which lets electron beam to hit the sample directly from the back. Cantilevers are glued on Ceramic holders by conductive UHV compatible epoxy, ${ }^{20}$ which are in turn held onto the cantilever holders by a leaf spring. Electrical wiring into the UHV system are introduced with two 20-pin 2.75 in. CF instrumentation feedthroughs. Kapton insulated 0.1 -mm-diam wires are used for all the electrical wiring to the microscope by carefully gluing with an UHV compatible conductive epoxy. ${ }^{20}$ Single mode fiber is introduced into UHV by a home-made fiber feedthrough. ${ }^{21} \mathrm{~A}$ small hole is drilled into a 1.33 in. CF blank flange, the fiber's plastic cladding is stripped before the fiber is fed through the hole and glued using Torr Seal. ${ }^{22}$

The cantilever is held fixed and the sample is scanned by using an EBL No. 2 piezotube ${ }^{23}$ in our microscope design as shown in Fig. 2. A piezoplate glued at the cantilever mounting block is used to vibrate the cantilevers. The cantilever displacements are measured with a novel all-fiber interferometer positioned accurately at the back of the cantilever with a piezodriven five-axis stick-slip slider. The scanner piezotube is mounted on another shear piezodriven stick-slip slider for coarse approach. Small, 1-mm-diam SiN balls are glued to the shear piezostacks ${ }^{24}$ fixed to the microscope base, which slide on polished sapphire plates attached to the sample slider block. Sample slider can move in $z$ and $y$ directions and rotate around the $x$ axis. Small $\mathrm{Sm}-\mathrm{Co}$ magnets and nickel sheets are used to pull the sample slider block towards the AFM base to increase the stiffness of the system. The bias voltage is applied to the cantilever and the tunneling current is measured from the sample with an in vacuum operational amplifier ${ }^{25}$ and a $100 \mathrm{M} \Omega$ feedback resistor, both placed just at the back of the scan tube. A stainless steel shield is mounted on the scan tube to minimize the capacitive coupling of high voltage scan signals to the tunnel current.

\section{Fiber slider}

We realized a high-precision and reliable five-axis positioner is required for the desired sensitivity levels of our microscope. The fiber is positioned at the back of cantilever by using a fiber slider, which has five degrees of freedom; three directions, $x, y, z$ and two rotations, $\theta$ and $\phi$. The fiber slider design, which is shown in Fig. 3, is similar to sample slider, but smaller in dimensions. Two piezodriven stick-slip sliders $(y z \phi$ and $x z \theta$ sliders), which can move in a plane and rotate around the axis normal to the plane are mounted on top of each other to form the fiber slider, which can move in five degrees of freedom. For the $y z \phi$ slider, which can move parallel to the microscope base and rotate, two shear plates are glued on top of each other with orthogonal polarization direction and then to the slider block. There are three sets of shear plates like this in the slider and 1-mm-diam SiN balls are glued to the piezoplates, which slide on polished sapphire plates fixed at the nc-AFM base. Since it is always more difficult to move against the gravity, three shear 


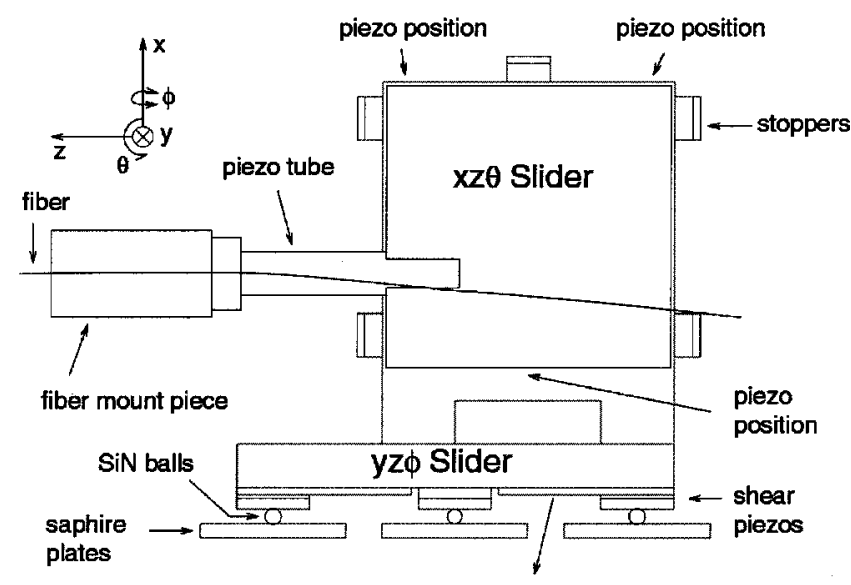

FIG. 3. Schematics of the fiber slider.

piezoplates are used in a stack for the $x z \theta$ slider: two for $x$, one for the $z$ direction. Three sets of piezostacks are glued on a steel L-shaped block, which is fixed on the $y z \phi$ slider. Polished sapphire plates are glued on the top piece of the $x z \theta$ slider. The fiber sliders are also loaded with the help of small $\mathrm{Sm}-\mathrm{Co}$ magnets and nickel plates to increase the stiffness of the slider. There are also stoppers on the base of $x z \theta$ slider to limit the slider motion. By applying appropriate pulses, one can move the sliders in five degrees of freedom with submicron resolution. The range of the fiber slider is $\pm 2, \pm 2$, and $\pm 8 \mathrm{~mm}$, and $\pm 5^{\circ}$ and $\pm 5^{\circ}$ in the $x, y, z, \theta$, and $\phi$ directions, respectively. The fiber is glued to steel holder attached at the end of a small piezotube, ${ }^{26}$ which is in turn glued to the top piece of the $x z \theta$ slider. This piezotube is used for fine positioning of the fiber with respect to the cantilever. The sliders are controlled using a joystick, while we monitor the motion with a long focal length video microscope.

\section{Fiber interferometer}

A novel all-fiber interferometer is designed for measuring the cantilever displacement with high measured sensitivity of $\sim 4 \times 10^{-4} \AA / \sqrt{\mathrm{Hz}}$. Our interferometer is similar to Rugar design, ${ }^{27}$ in principle, with a couple of tricks to improve the sensitivity. A fiber pigtailed, laser diode operated at constant power is coupled into a $2 \times 2$, single mode, $50 \%$ fiber splitter as shown in Fig. 4. One of the fiber splitter outputs is connected to the fiber, which goes into the UHV system and is positioned very close to the back of cantilever. The other output and the input of the single mode splitter are connected to pigtailed reference and signal photodiodes, respectively. The laser output is divided into two at the fiber splitter and the part of the light incident to the fiber is reflected at the fiber end. The rest of the light exits the fiber, hits the cantilever and is reflected back into the fiber. These two beams reach to the signal photodiode after passing the fiber splitter. Interference of these beams generates a photocurrent at the signal photodiode. Output of the reference photodiode on the other hand is used to monitor the laser power and is subtracted from the output of signal photodiode to compensate for the laser power fluctuations and laser intensity noise. The end of the fiber is first cleaved with a precision fiber cleaver and then coated with $\mathrm{Si}$ and $\mathrm{Au}$ to enhance
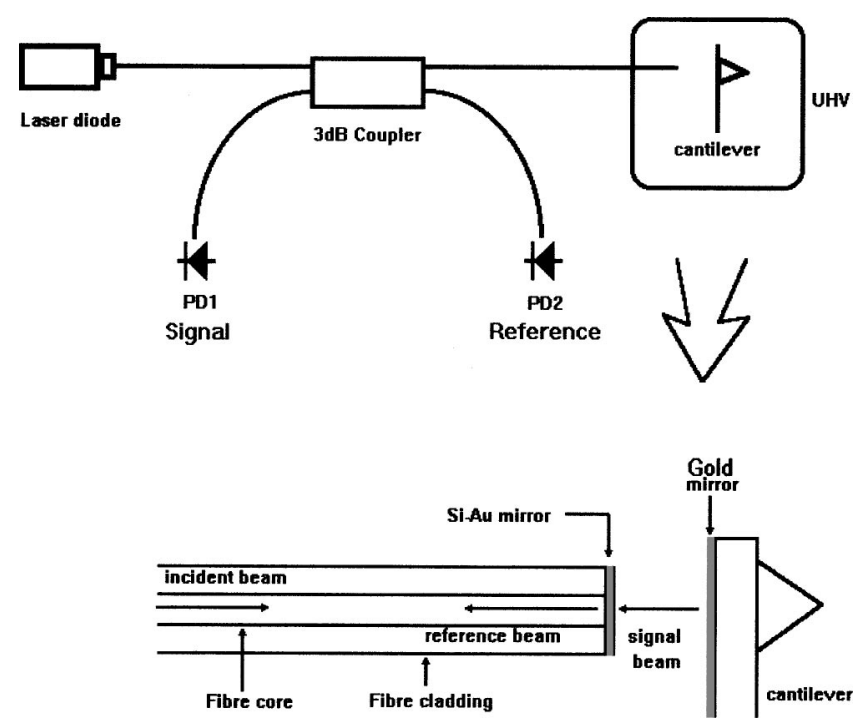

FIG. 4. All-fiber interferometer.

the reflectivity. If the fiber is positioned parallel and very close to the lever, a Fabry-Pérot cavity is formed, and one can get multiple reflections between the back of the cantilever and the fiber end, which will in turn enhance the sensitivity of interferometer. However, one needs to adjust the relative angle of the fiber with respect to cantilever accurately to get multiple reflections.

When the fiber is not parallel to the lever or far away from the lever, there are no multiple reflections and the signal photodiode current can be written as ${ }^{27}$

$$
i=i_{0}[1-V \cos (4 \pi d / \lambda)],
$$

where $V, d$, and $\lambda$ are visibility, fiber-lever separation, and the wavelength of the laser. This is a typical Michaelson interferometer and at the quadrature points, $d=\lambda / 8,3 \lambda / 8$, $5 \lambda / 8, \ldots$, where the interferometer is at the most sensitive position, the slope of the interferometer is

$$
\Delta i / \Delta d=4 \pi i_{0} V / \lambda,
$$

where $\Delta d$ is cantilever displacement. When our interferometer is well aligned and the fiber is parallel to the lever, the signal photodiode current can be written as

$$
i=i_{0}[1-V F(4 \pi d / \lambda)],
$$

where $F(d)$ is a periodic function of $d$, as shown in Fig. 5, which has a much sharper slope, $m=F^{\prime}(d)$, than the Michaelson interferometer at the proper quadrature point. Therefore, the slope of the interferometer at a quadrature point can be written as

$$
\Delta i / \Delta d=4 \pi i_{0} V m / \lambda \text {. }
$$

The slope of this function $F(d), m$, is always greater than 1 at the proper quadrature point. The other quadrature point usually has a slope less than 1 . Typical values we can get are $m=4-5.5$ and $V=0.3-0.8$ by carefully aligning the fiber. A typical interference pattern is shown in Fig. 5. The smallest displacement that can be measured by our interferometer will be limited by the shot noise, which can be written as ${ }^{27}$

$$
d_{\text {shot }}=(\lambda / 2 \pi V m) \sqrt{\left(e f / 2 S P_{\mathrm{op}}\right)},
$$




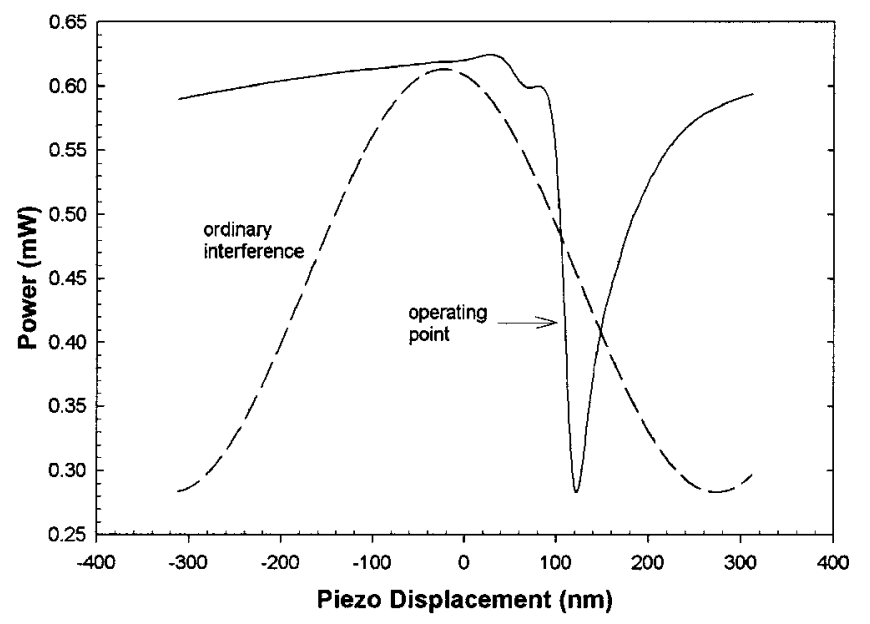

FIG. 5. Typical interference pattern.

where $e, f, S$, and $P_{\text {op }}$ are electron charge, bandwidth of measurement, photodiode responsivity, and the power at the quadrature point, respectively. For typical operating parameters of $\lambda=1320 \mathrm{~nm}, V=0.39, m=5.5, S=1 \mathrm{~A} / \mathrm{W}$, and $P_{\mathrm{av}}$ $=400 \mu \mathrm{W}$, our shot noise limit is $\sim 1.4 \times 10^{-5} \AA / \sqrt{\mathrm{Hz}}$. Figure 6 shows the noise spectrum of our fiber interferometer with a measured noise level of $\sim 4 \times 10^{-4} \AA / \sqrt{\mathrm{Hz}}$. This is considerably higher than the shot noise limit, which can be improved further. Operating at high slopes does not come without any cost; the high slope is only sustained for a narrow region of fiber-cantilever separations and is susceptible to thermal drifts. We employ a special feedback loop implemented in the software to overcome this problem. This simple feedback loop keeps the interferometer always at the proper quadrature position by moving the fiber using the fiber piezotube. A high power video microscope guides the initial fiber-lever adjustment with a $\times 40$ magnification, while moving the five-axis fiber slider with joystick control. After getting some reflection from the lever under the optical microscope guidance, the interference pattern itself is used as a guide and the best fiber position is found with the fiber slider.

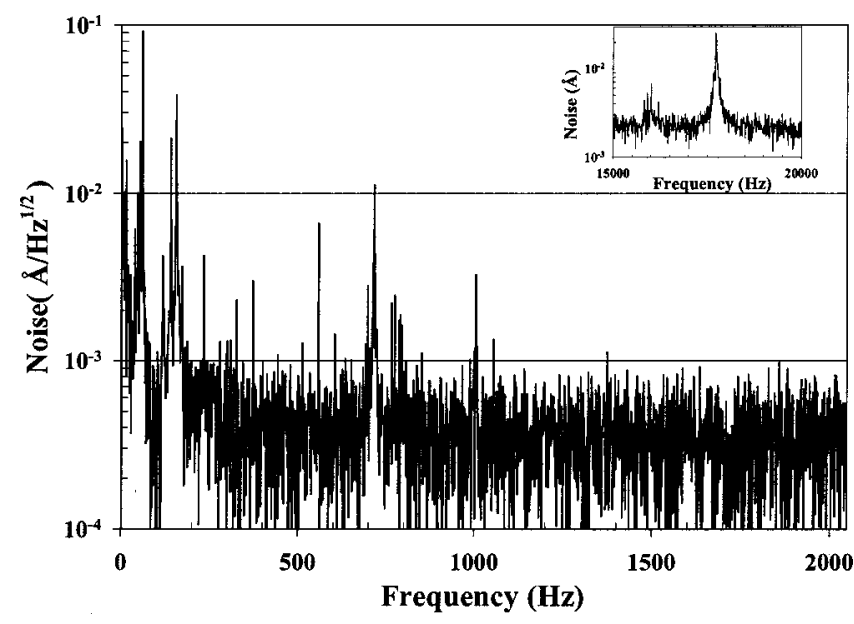

FIG. 6. Noise spectrum of the fiber interferometer obtained with a tungsten cantilever in UHV.

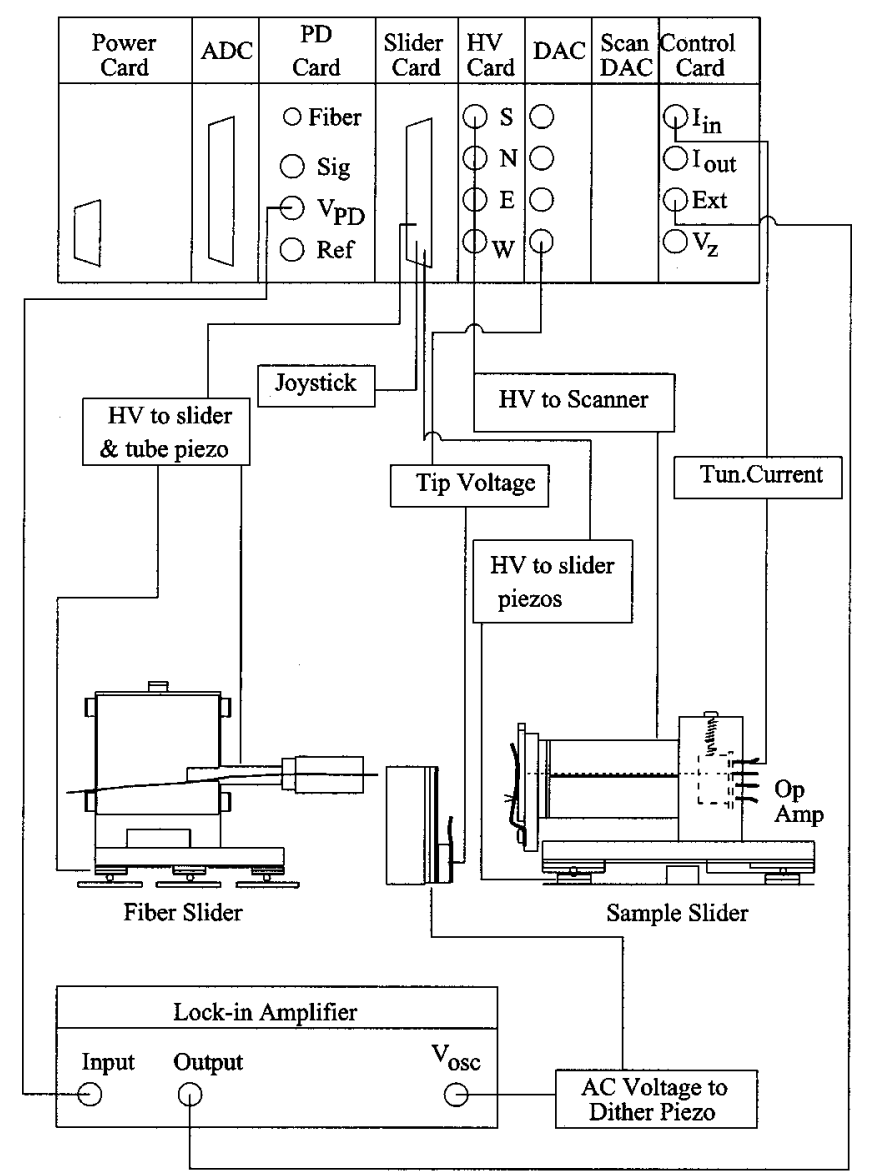

FIG. 7. Schematic diagram of the electronics.

\section{Electronics and software}

The nc-AFM/STM is controlled by a PC through a custom-built electronics ${ }^{28}$ and software, as shown in Fig. 7. The electronics consists of nine PCB cards. The high voltage amplifier card is used to combine $X$ and $Y$ scan signals to $V_{z}$ to drive the scan piezoquadrants. The slider card supplies the voltage pulses to the sliders' shear piezos. The photodetector card incorporates the laser diode, automatic power control circuit, the fiber coupler, two photodetectors, and the relevant amplifiers. Two analog-to-digital converter cards can sample a total of 16 analog signal channels with 16 bit resolution at $200 \mathrm{kHz}$ through a software programmable gain amplifier with gains of 1, 2, 4, and 8. A digital-to-analog converter card supplies various control voltages with 16 bit resolution. Scan generator card has two 20 bit digital to analog converters for $X$ and $Y$ scan voltages. The feedback control card, which regulates the tip-sample separation, can be configured for STM, AFM, or external input feedback. All the functions of the electronics are controlled through the software specially developed for this project. There are no manual control inputs, all the adjustments of the microscope are performed with the software. The program performs automatic sample approach, image acquisition, force-distance curve, interference pattern measurements, quadrature locking for staying at highest sensitivity point, image display, and various postacquisition image manipulations. 


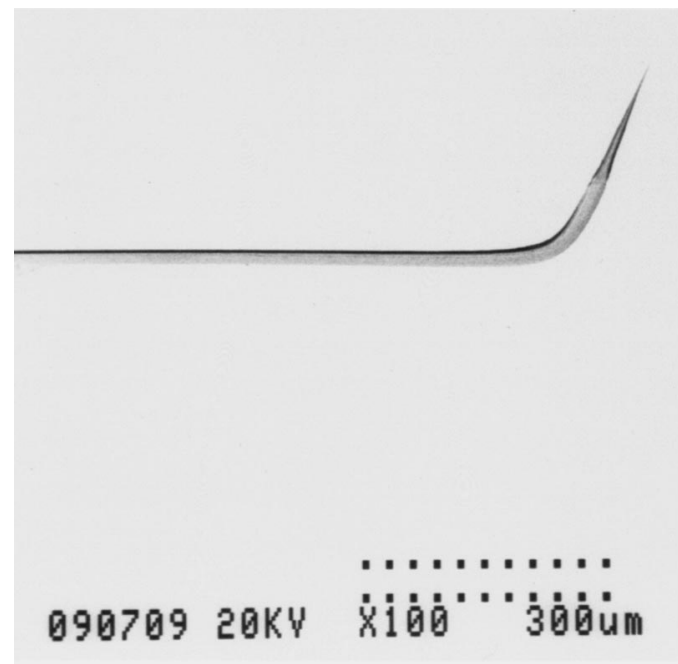

FIG. 8. Scanning electron microscopy image of a tungsten lever.

\section{Operation of the microscope}

The cantilever and piezomotions have been calibrated by the fiber interferometer in $z$ direction and with STM imaging of $\operatorname{Si}(111)(7 \times 7)$ surface in the $X Y$ direction. The microscope is stable for long periods of time and we can even log images overnight automatically. The cantilevers are made from flattened and polished tungsten wires whose ends are bent and then etched to obtain sharp tips as shown in Fig. 8. The cantilevers' back are coated with a 50-100-nm-thick gold layer to increase the reflectivity. The levers have stiffnesses typically in the range of $50-250 \mathrm{~N} / \mathrm{m}$, with $11-20 \mathrm{kHz}$ resonant frequencies and a typical $Q$ of 3000 in UHV. The stiffness of the levers is determined by measuring the thermal oscillation amplitude and independently by a nanoindenter. No other special tip preparation is performed apart from baking the whole chamber with the levers and the sample to achieve the base pressure of $\sim 7 \times 10^{-11}$ mbar in the system. Even though the tip is tungsten to start with, it is highly likely that we pick up a number of silicon atoms from the surface as we start to perform our experiments. Atomically clean $\mathrm{Si}(111)(7 \times 7)$ is prepared in situ by flashing the sample to $1000^{\circ} \mathrm{C}$. The force gradient between tip and sample is measured by vibrating the cantilever at a frequency well below the resonance, with a subangstrom oscillation amplitude. Changes in oscillation amplitude due to the tip-surface interaction are then detected using a lock-in amplifier. For small interaction forces and oscillation amplitudes, the interaction force gradient between tip and sample, $f^{\prime}$, can be obtained a from the simple relation ${ }^{29}$

$$
f^{\prime}=k_{0}\left(A_{0} / A-1\right),
$$

where, $k_{0}, A_{0}$, and $A$ are the lever stiffness, free oscillation amplitude, and measured oscillation amplitude of the lever, respectively. During imaging, the changes in the oscillation amplitude of the lever are acquired by using a lock-in amplifier simultaneously with the STM topography. These data are then processed to obtain the force gradient image, using Eq. (6). The important feature is that we are able to use very small amplitudes, with $0.125-0.25 \AA$ being typical for the data in this paper. Therefore, even strongly varying forces

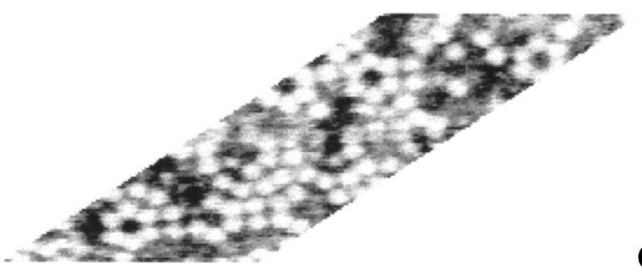

(a)

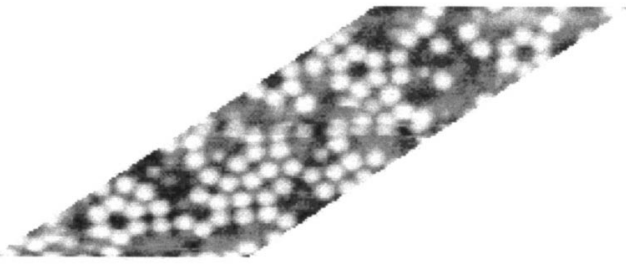

(b)

FIG. 9. Simultaneous force gradient image (a) and STM image (b) of $\operatorname{Si}(111)(7 \times 7)$. Measurement conditions: $V_{\text {tip }}=-2 \mathrm{~V}, I_{T}=0.1 \mathrm{nA}, A_{0}$ $=2 \AA$, and $k_{0}=116 \mathrm{~N} / \mathrm{m}$. Black shows more negative values in the force gradient image.

can be simply measured, without concern about the overall nonlinearity of the interaction potential, which affects larger amplitude oscillation technique.

\section{RESULTS AND DISCUSSIONS}

The initial experiments are performed with relatively large oscillation amplitude of $2 \AA$. Figure 9 shows the force gradient and STM image of a $\mathrm{Si}(111)(7 \times 7)$ sample acquired simultaneously. There are a large number of defects on the surface, because the images have been acquired long time after sample cleaning. Since we use a lock-in amplifier to detect the cantilever displacements, we have to use relatively slow scan speeds of $10-30 \AA / s$, which makes images quite prone to the thermal drift.

The oscillation amplitudes are then decreased to $0.25 \AA$ to measure the force gradient-distance $\left(f^{\prime}-d\right)$ curves. We freeze the feedback, pull the sample away from the cantilever, and then start to approach the sample towards the cantilever as we monitor the tunnel current and the force gradient simultaneously to measure the force interactions between the tip and sample. Along the approach curve we stop at a predetermined tunnel current, force, or force gradient value, and then retract the sample as we record the same quantities. Much of the time we see longer range force interactions and the forces are already significant before any tunnel current is observed, as shown in Fig. 10. This must be due to presence of longer-range forces such as van der Waals or electrostatic, which because of either tip shape or chemical nature of tip material, outweigh and thus mask the short range, highly local forces. However, with other tips we observe behavior such as that shown in Figs. 10 and 11. The tunnel current in these cases rises at or before the onset of an observable force interaction, an indication that the longer-range forces are not dominant. The tunnel barrier $\Phi=d \ln I / d z$ in Fig. 11, starts from $\sim 2 \mathrm{eV}$ at larger separations and drops, as is expected ${ }^{30}$ in the stronger interaction region when the tip approaches close into the surface. The whole approach and retraction process is reversible provided the separation is not reduced beyond the point at which the stiffness again becomes positive (the point of inflection of the binding energy curve). The 


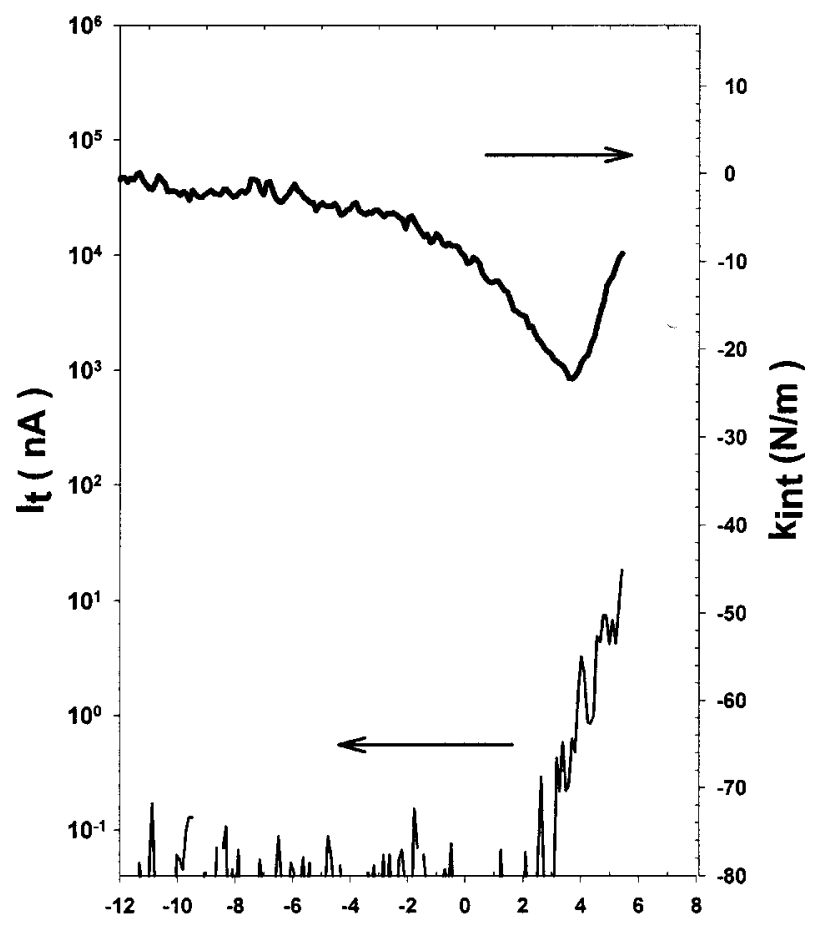

Sample Displacement ( Angstrom )

FIG. 10. Force gradient and tunnel current as a function of tip-sample separation. Typical result for tips having longer range interaction. The length scale of the interaction is $2.2 \AA \pm 0.4 \AA . A_{0}=0.25 \AA$, and $k_{0}=90 \mathrm{~N} / \mathrm{m}$.

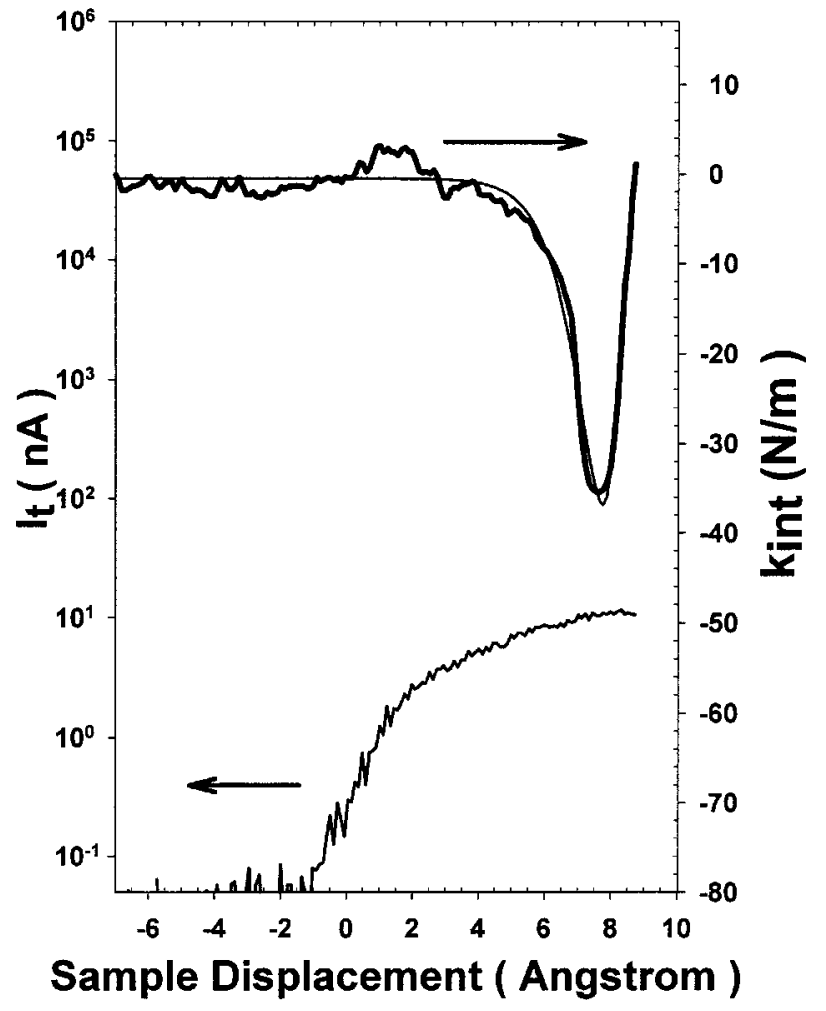

FIG. 11. Force gradient and tunnel current as a function of tip-sample separation. Typical result for tips having short-range interaction. The length scale of the interaction is $0.75 \AA \pm 0.2 \AA$ found from the fit (thin line). $A_{0}$ $=0.25 \AA$ and $k_{0}=250 \mathrm{~N} / \mathrm{m}$.

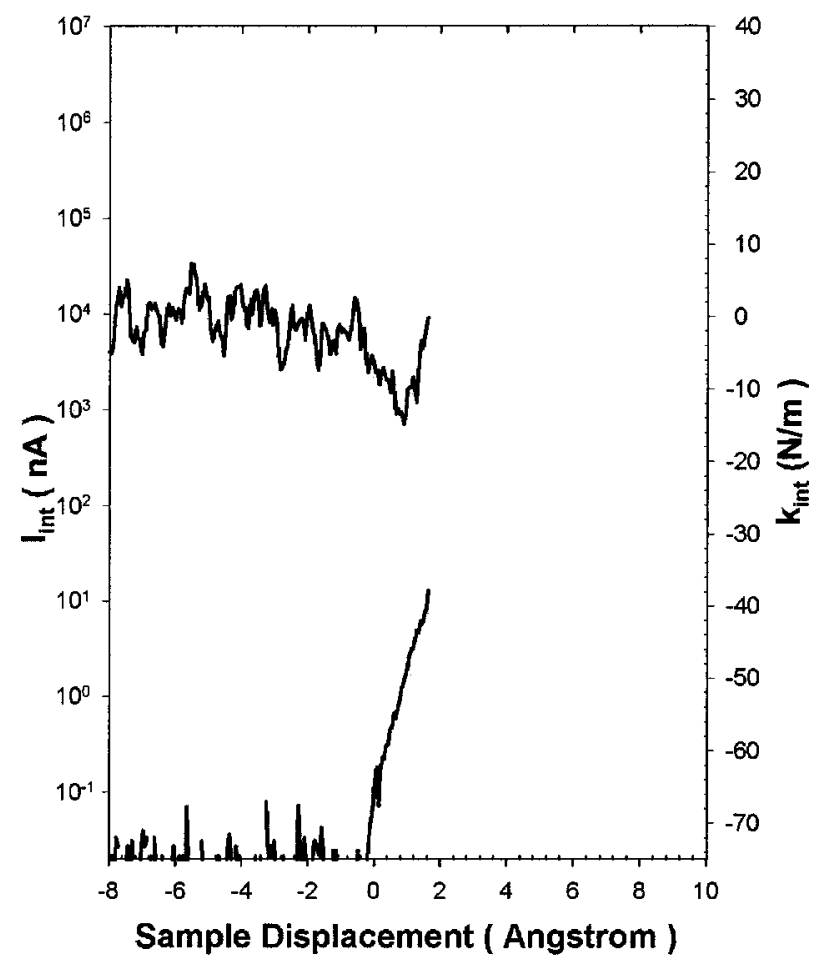

FIG. 12. Force gradient and tunnel current as a function of tip-sample separation. Typical result for tips having short-range interaction. The short range and magnitude of gradient indicate this is mostly due to a single atomic bond. $A_{0}=0.125 \AA$ and $k_{0}=250 \mathrm{~N} / \mathrm{m}$.

curves shown here are all single approach curves in contrast to large oscillation amplitude $\Delta f-d$ curves where the measurement is averaged over thousands of approach-retract cycle. Most importantly, we can fit our measured $f^{\prime}-d$ curves to a universal bonding potential ${ }^{31}$ and we obtain a decay length scale for the tip interaction of $2.2 \AA \pm 0.4 \AA$ for Fig. 9 and $0.75 \AA \pm 0.2 \AA$ for Fig. 10. This agrees well with theoretical expectations for bonding, and is unlike previous experiments where values $3 \AA$ or more are reported. ${ }^{9,10}$ This indicates that we are really measuring the short-range potentials responsible for strong interatomic bonds. We shall see next that this is confirmed in the force gradient imaging. The $f^{\prime}-d$ curve in Fig. 12 has a maximum tensile stiffness of $\sim 13 \mathrm{~N} / \mathrm{m}$ and the integration of this gives $\sim 3 \mathrm{nN}$ maximum force, which is in perfect agreement with the ab initio calculations. ${ }^{11}$ While most of the interaction is due to a single pair of atoms, on tip and on surface, some extra, smaller, interactions with immediately adjacent atoms should also be present. The excess noise in the $f^{\prime}-d$ curve displayed in Fig. 12 is due to much lower, $0.125 \AA$, oscillation amplitudes as well as a small time constant used in the lock-in amplifier that we normally use.

After getting some images using a relatively large oscillation amplitude of $2 \AA$ to test the system, we have reduced the oscillation amplitude down to $0.25 \AA$ to obtain images of bond stiffness variations across the $\operatorname{Si}(111)(7 \times 7)$ surface. Figure 13 shows force gradient and STM topography images obtained simultaneously on a $\mathrm{Si}(111)(7 \times 7)$ sample. A crucial 


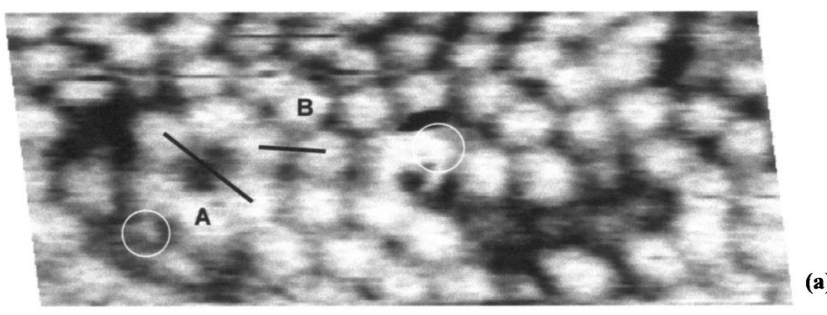

(a)

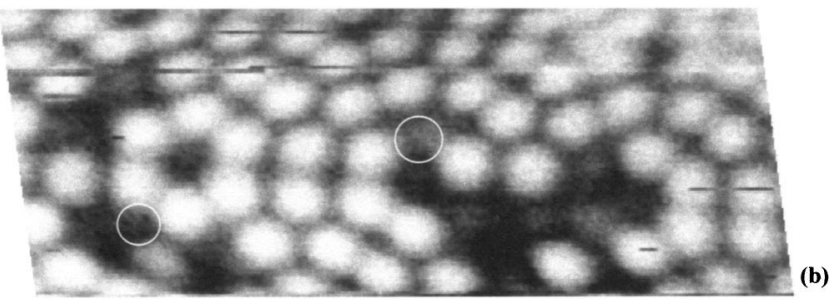

FIG. 13. Simultaneous force gradient image (a) and STM images (b) of $\mathrm{Si}(111)(7 \times 7)$ sample where the tip changes half way up the image. $V_{\text {tip }}=$ $-1 \mathrm{~V}, I_{T}=2 \mathrm{nA}, A_{0}=0.25 \AA$, and $k_{0}=116 \mathrm{~N} / \mathrm{m}$. Black shows more negative values in the force gradient image.

point is that we were only able to obtain atomic resolution in the force images, such as shown in Fig. 9, when the tips also gave short-range interactions such as that shown in Figs. 11 and 12 . This clearly supports the traditional supposition that atomic resolution in AFM requires a rapid exponential interaction, hence sharp tips. Bond stiffness images such as Fig. 13 give direct quantitative data. First of all, it should be noted that STM topography and force gradient images, although broadly similar, do not show exactly the same features. For example, the areas circled in Fig. 13 show that the missing adatoms in the STM image give a different contrast in AFM image, perhaps due to an adsorbate on the surface. We find that the corrugation from adatom to adatom via corner hole (line A) and from adatom to adatom via restatom (line B) are 12 and $4.5 \mathrm{~N} / \mathrm{m}$, respectively. The black shows higher attractive stiffness in the force gradient images, and therefore, the corner holes and restatoms actually have higher attractive stiffness than the adatoms. The force gradient contrast is also seen to increase with decreasing separation at higher tunnel currents. Similar behavior has also been observed on the $\mathrm{Si}(100)(2 \times 1)^{32}$ surface in our recent experiments.

Differing tips give an even more complex relationship, as images such as Fig. 14 show. The tip changes half way up the image, resulting in a much reduced STM contrast in the upper half. But, this same tip change actually increases most of the contrast in the simultaneous force gradient image. The corrugation from adatom to adatom via corner hole (line A) decreases from $11 \mathrm{~N} / \mathrm{m}$ before the tip change, to $8.4 \mathrm{~N} / \mathrm{m}$ afterwards, as shown in Fig. 14. Interestingly, however, the corrugation from adatom to adatom via restatom (line B) increases from 5.2 to $6.9 \mathrm{~N} / \mathrm{m}$. The adatom-restatom force corrugation is increased by the tip change but the adatomcorner hole corrugation is decreased. There is obviously fertile ground here for study of the detailed configuration of individual surface bonds. ${ }^{14}$

We have shown that, using ultrasmall oscillation amplitudes, atom-resolved quantitative AFM imaging, and direct

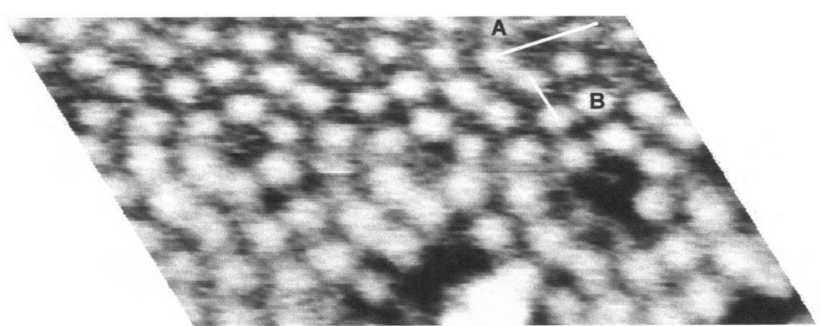

(a)

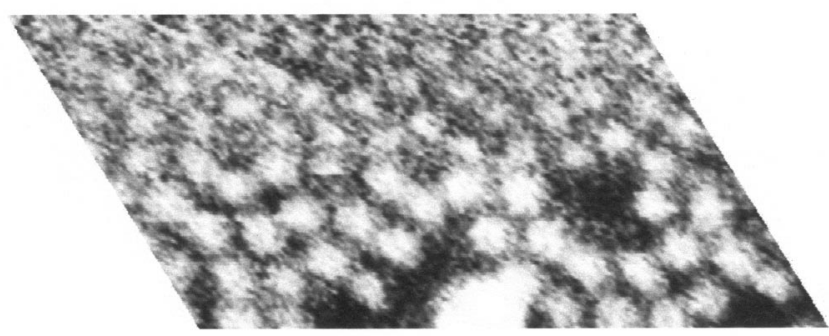

(b)

FIG. 14. Simultaneous force gradient image (a) and STM image (b) of $\mathrm{Si}(111)(7 \times 7)$. Measurement conditions: $V_{\text {tip }}=-1 \mathrm{~V}, I_{T}=1 \mathrm{nA}, A_{0}$ $=0.25 \AA$, and $k_{0}=116 \mathrm{~N} / \mathrm{m}$. Black shows more negative values in the force gradient image.

force spectroscopy are possible. Short-range interactions are crucial for imaging, but long-range terms need to be incorporated to explain the image corrugations observed. ${ }^{14,32,33}$ This novel method can also be employed to measure the forces involved in atomic manipulations. ${ }^{34}$ Moreover, our technique is not limited to UHV only and operation under liquids ${ }^{15}$ and air is feasible.

\section{ACKNOWLEDGMENTS}

This work is supported by the Paul Fund and EPSRC in the U.K. One of the authors (A.O.) thanks the British Council for travel support and a second author (H.Ö.Ö) thanks The Scientific and Technical Research Council of Turkey (TÜBITAK) for a BDP scholarship.

${ }^{1}$ G. Binnig, C. F. Quate, and Ch. Gerber, Phys. Rev. Lett. 56, 930 (1986).

${ }^{2}$ C. J. Chen, Introduction to Scanning Tunneling Microscopy (Oxford University Press, Oxford, UK, 1993), pp. 185-193.

S. Ciraci, in Scanning Tunneling Microscopy, edited by R. Wiesendanger and H.-J. Guntherodt (Springer, Berlin, 1996), Vol. 3, Chap. 8.

${ }^{4}$ F. J. Giessibl, Science 267, 69 (1995).

${ }^{5}$ H. Ueyama, M. Ohta, Y. Sugawara, and S. Morita, Jpn. J. Appl. Phys., Part 2 34, L1086 (1995).

${ }^{6}$ S. Kitamura and M. Iwatsuki, Jpn. J. Appl. Phys., Part 2 34, L145 (1995).

${ }^{7}$ M. A. Lantz, H. J. Hug, P. J. A. van Schendel, R. Hoffmann, S. Martin, A. Baratoff, A. Abdurixit, H.-J. Güntherodt, and Ch. Gerber, Phys. Rev. Lett. 84, 2642 (2000)

${ }^{8}$ U. Dürig, O. Züger, and D. W. Pohl, Phys. Rev. Lett. 65, 349 (1990).

${ }^{9}$ G. Cross et al., Phys. Rev. Lett. 80, 4685 (1998).

${ }^{10}$ S. P. Jarvis, H. Yamada, S.-I. Yamamoto, H. Tokumoto, and J. B. Pethica, Nature (London) 384, 247 (1996)

${ }^{11}$ R. Perez, M. C. Payne, I. Stich, and K. Terakura, Appl. Surf. Sci. 123, 249 (1998)

${ }^{12}$ H. Ueyama, Y. Sugawara, and S. Morita, Appl. Phys. A: Mater. Sci. Process. A66, 295 (1998).

${ }^{13}$ R. Lüthi et al., Z. Phys. B: Condens. Matter 100, 165 (1996).

${ }^{14}$ A. Oral, R. A. Grimble, H. Ö. Özer, P. M. Hoffmann, and J. B. Pethica, Appl. Phys. Lett. 79, 1915 (2001).

${ }^{15}$ S. Jeffery, Ph.D. thesis, Oxford University (2001). 
${ }^{16}$ Omicron NG LEED system with SpectaLEED controller.

${ }^{17}$ Varian 300 Combo Pump, Varian, Inc.

${ }^{18}$ Varian V-70LP Turbomolecular Pump. Varian, Inc.

${ }^{19}$ WS75 Pincer-grip Wobble stick, Vacuum Generators, Ltd., Hastings, UK.

${ }^{20}$ EpoTek H21D, Epoxy Technology, Inc.

${ }^{21}$ Y. Sugawara (private communication).

${ }^{22}$ Torr Seal, UHV compatible seal, Varian, Inc.; this seal works up to $100{ }^{\circ} \mathrm{C}$. Therefore, we water cool the fiber feedthrough flange during bakeout to $100^{\circ} \mathrm{C}$.

${ }^{23}$ EBL No. 2 piezotube, $12.7 \mathrm{~mm}$ OD $\times 1 \mathrm{~mm}$ wall thickness, $25.4 \mathrm{~mm}$ long, Staveley Sensors, Inc., 91 Prestige Circle, East Hartford, CT.

${ }^{24}$ EBL No. 2 shear plates, Staveley Sensors, Inc., 91 Prestige Circle, East Hartford, CT.

${ }^{25}$ OPA111AM OP-AMP, Texas Instruments.

${ }^{26}$ EBL No. 1 piezotube, $3.18 \mathrm{~mm}$ OD $\times 0.38 \mathrm{~mm}$ wall thickness, $12.7 \mathrm{~mm}$ long, Staveley Sensors, Inc., 91 Prestige Circle, East Hartford, CT.
${ }^{27}$ D. Rugar, H. J. Mamin, and P. Guethner, Appl. Phys. Lett. 55, 2588 (1989).

${ }^{28}$ The electronics and the software are now available from NanoMagnetics Instruments, Ltd.; www.nanomagnetics-inst.com

${ }^{29}$ S. P. Jarvis, A. Oral, T. P. Weihs, and J. B. Pethica, Rev. Sci. Instrum. 64, 3515 (1993).

${ }^{30}$ U. Dürig, J. K. Gimzewski, and D. W. Pohl, Phys. Rev. Lett. 57, 2403 (1986).

${ }^{31}$ A. P. Sutton, Electronic Structure of Materials (Oxford University Press, Oxford, U.K., 1993), pp. 1-6.

${ }^{32}$ H. Ö. Özer, M. Atabak, R. Ellialtioğlu, and A. Oral, Appl. Surf. Sci. (in press).

${ }^{33}$ P. M. Hoffmann, R. A. Grimble, J. B. Pethica, H. Ö. Özer, and A. Oral, Proc. R. Soc. London, Ser. A 457, 1161 (2001).

${ }^{34}$ T. W. Fishlock, A. Oral, R. G. Egdell, and J. B. Pethica, Nature (London) 404, 743 (2000). 